Gefässchirurgie 2020 • 25:91-104 https://doi.org/10.1007/s00772-020-00616-5 Online publiziert: 18 . Februar 2020

(c) Der/die Autor(en) 2020

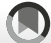

Check for
updates

W. Schäberle

Abteilung für Viszeral-, Thorax- und Gefäßchirurgie, Alb-Fils-Kliniken, Göppingen, Deutschland

\title{
Sonographische Graduierung von Karotisstenosen
}

\section{Einflussgrößen auf die hämodynamische Stenosegraduierung}

tionen (Stent, TEA, EEA) akzeptiert. Neben Strahlenbelastung, Kontrastmittelexposition und Komplikationen durch den invasiven Gefäßzugang (Aneurysma spurium und Dissektion) wurde damals eine hohe Anzahl (in 1 bis 4\%) an zerebrovaskulären Ereignissen (wie TIA’s) im Rahmen der diagnostischen Angiographie publiziert $[10,11]$. In den ESVSLeitlinien wird die ACAS-Studie mit zerebrovaskulären Ereignissen in 1,3\% bei Angiographien zitiert [12]. Deshalb wird von Angiographien abgeraten und die FKDS als primäre Methode in der Diagnostik empfohlen [12].

》) Die FKDS wird als primäre Methode in der Diagnostik empfohlen

\section{Hintergrund}

Aufgrund einer Vielzahl von Studien [1-8], in denen die Sonographie (FKDS) eine Treffsicherheit von um die $90 \%$ gegenüber dem bisherigen ,golden standard“ Angiographie (i.a. DSA) zeigte, hat sich die duplexsonographische Stenosegraduierung bei Karotisstenosen zur Therapieplanung durchgesetzt und etabliert. Auswertungen von Angiographieuntersuchungen durch zwei unterschiedliche Radiologen zeigen auch keine höhere Übereinstimmung [9]. Sowohl aufgrund der hohen Treffsicherheit der nichtinvasiven FKDS als auch aufgrund der Komplikationen der invasiven DSA-Untersuchung ist die sonographische Stenosegraduierung als Grundlage zur Therapieplanung auch vor Interven-
Hauptkriterium der hämodynamischen Stenosegraduierung durch die Duplexsonographie (FKDS) ist die intrastenotische, systolische Spitzengeschwindigkeit (PSV). Sie wird in angloamerikanischen Studien oft als ausschließliches Stenosekriterium angewandt. Die Deutsche Gesellschaft für Ultraschall in der Medizin (DEGUM) hat demgegenüber den Einsatz weiterer Zusatzkriterien gefordert. Zurecht wird jedoch die unterschiedliche Auswahl verschiedener Stenosekriterien, wie auch die Diskrepanz in den Stenosekriterien und der PSV-Grenzgeschwindigkeit in unterschiedlichen Studien beklagt [13].

Die PSV ist von verschiedenen systemischen Faktoren abhängig. Hierzu gehören der systolische Blutdruck, die
Gefäßwandrigidität, die Kollateralfunktion bei bilateraler Karotisstenose sowie vorgeschaltete (Aortenklappenstenose) und nachgeschaltete, intrakranielle Stenosen. Daher wurde durch die DEGUM eine multiparametrische Auswertung mit Haupt- und Zusatzkriterien (- Tab. 1) zur genauen Stenosegraduierung und zur Vergleichbarkeit der Untersuchungsergebnisse (Verbesserung der Interobserver- wie auch Intraobservervarianz) eingeführt [14]. Dennoch gibt es immer wieder Studien, die die Duplexsonographie mit präinterventioneller Stenosegraduierung als die therapieplanende Untersuchungsmethode in Zweifel ziehen. In einer jüngst publizierten Studie wurde nicht nur die PSV, sondern erstmals die multiparametrischen Ultraschallkriterien der DEGUM gegenüber der Angiographie validiert [15]. Dennoch wurde resümiert, dass in der Identifikation von operationsrelevanten Stenosegraden der A. carotis interna (ICA) die Anwendung der multiparametrischen DEGUM-Ultraschallkriterien nicht die Notwendigkeit eines „vaskulären Bestätigungstestes“ wie einer Angiographie als Kontrolluntersuchung beseitigen würde.

\section{Geschichte der sonographischen Stenosegraduierung}

Bis 2010 beurteilten die angloamerikanischen und die europäischen Studien den Stenosegrad nach unterschiedlichen Messmethoden: Angloamerikanische Studien, wie auch die NASCET-Studie $[16,17]$, verwendeten den distalen Ste- 
Tab. 1 Duplexsonographische Kriterien zur Graduierung von Stenosen der A. carotis interna [2, 14, 22, 28]

\begin{tabular}{|c|c|c|c|c|c|c|c|c|c|}
\hline \multicolumn{2}{|c|}{$\begin{array}{l}\text { Distaler Stenosegrad } \\
\text { (NASCET-Definition in \%) }\end{array}$} & 10 & $20-40$ & 50 & 60 & 70 & 80 & 90 & Verschluss \\
\hline \multicolumn{2}{|c|}{$\begin{array}{l}\text { Lokaler Stenosegrad } \\
\text { (ECST-Definition in \%) }\end{array}$} & 40 & $50-60$ & 70 & 75 & 80 & 90 & 95 & Verschluss \\
\hline \multirow[t]{5}{*}{ Hauptkriterien } & 1. B-Bild & +++ & + & & & & & & \\
\hline & 2. Farb-Doppler-Bild & + & +++ & + & + & + & + & + & +++ \\
\hline & $\begin{array}{l}\text { 3. Systolische Spitzengeschwindigkeit } \\
\text { (PSV) im Stenosemaximum [cm/s] ca. }\end{array}$ & $<100$ & $120-160$ & $\begin{array}{l}200 \\
\left(125^{* a}\right)\end{array}$ & 250 & $\begin{array}{l}300 \\
\left(230^{* a}\right)\end{array}$ & $350-400$ & $200-500$ & $\begin{array}{l}\text { Kein } \\
\text { Signal }\end{array}$ \\
\hline & $\begin{array}{l}\text { 4. Systolische Spitzengeschwindigkeit } \\
\text { poststenotisch }[\mathrm{cm} / \mathrm{s}]\end{array}$ & & & & & $>50$ & $<50$ & $<30$ & $\begin{array}{l}\text { Kein } \\
\text { Signal }\end{array}$ \\
\hline & $\begin{array}{l}\text { 5. Kollateralen und Vorstufen (Periorbi- } \\
\text { talarterien) }\end{array}$ & & & & & $(+)$ & ++ & +++ & +++ \\
\hline \multirow[t]{5}{*}{ Zusatzkriterien } & $\begin{array}{l}\text { 6. Diastolische Strömungsverlangsa- } \\
\text { mung prästenotisch (CCA) }\end{array}$ & & & & & $(+)$ & ++ & +++ & +++ \\
\hline & 7. Strömungsstörungen poststenotisch & & & + & + & ++ & +++ & $(+)$ & \\
\hline & $\begin{array}{l}\text { 8. Enddiastolische Strömungsgeschwin- } \\
\text { digkeit (EDV) im Stenosemaximum } \\
{[\mathrm{cm} / \mathrm{s}] \mathrm{ca} \text {. }}\end{array}$ & & & $<100$ & $<100$ & $>100$ & $>100$ & & \\
\hline & 9. Konfettizeichen & & & & $(+)$ & ++ & ++ & & \\
\hline & 10. Stenoseindex ICA/CCA & & & $\geq 2$ & $\geq 3$ & $\geq 4$ & $\geq 4$ & & \\
\hline
\end{tabular}

nosegrad. Dagegen wurde in der ECSTStudie [18] nach dem insbesondere auch von der Deutschen Gesellschaft für Ultraschall in der Medizin propagierten lokalen Stenosegrad graduiert. Der angiographisch leichter zu vermessende distale Stenosegrad, bei dem das intrastenotische Restlumen mit dem Normallumen der distalen unauffälligen A. carotis interna (NASCET-Kriterium) verglichen wird (• Abb. 1), wäre die adäquate Stenosegradbestimmung, wenn primär eine stenosebedingte, globale Durchblutungsminderung im Vordergrund stehen würde. Die Problematik der Karotisstenose (jedenfalls, wenn sie nur unilateral höhergradig auftritt) liegt jedoch nicht primär in der Durchblutungsreduktion für das Gehirn, sondern in intrakraniellen Embolien aus stenosierenden ICA+Plaques. Da die Emboliegefahr vor allem auch durch die Plaquedicke getriggert wird, wird diese im lokalen Stenosegrad, der das intrastenotische Restlumen mit dem Bulbuslumen in Relation (ECST-Kriterium) setzt, adäquater ausgedrückt. Im zur differenzierten Therapieplanung relevanten Stenosesegment von 40 bis $80 \%$ ergeben sich dabei Diskrepanzen von bis zu $30 \%$ (• Abb. 1, • Tab. 1).
Bedingt durch die unterschiedliche Messmethodik in der Stenosegraduierung sind Studien, wie auch die großen Multicenterstudien NASCET [17] und ECST [18] schwer vergleichbar. Daher wurde nach einer Konsensuskonferenz 2010 die PSV als wichtigstes Stenosekriterium an die NASCET-Kriterien angeglichen und eine PSV von $200 \mathrm{~cm} / \mathrm{s}$ von einer 70\%igen Stenose nach lokalem Stenosegrad (ECST) zu einer 50\%igen Stenose (distaler Stenosegrad, NASCETKriterium) umgewertet. Gleichzeitig wurden nochmals die von der DEGUM zur adäquaten Stenosegraduierung entworfenen zusätzlichen Haupt- und Nebenkriterien festgeschrieben und durch die Neurosonology Research Group of the World Federation of Neurology angenommen [19].

Parallel zu diesem Diskussionsprozess mit Angleichung der europäischen an die angloamerikanischen Stenosekriterien wurde in den USA durch weitere Vergleichsstudien mit der Angiographie die PSV-Grenzgeschwindigkeit nach NASCET-Kriterium (distaler Stenosegrad) für eine $>50 \%$ ige Stenose von 200 auf $130 \mathrm{~cm} / \mathrm{s}$ und für eine $>70 \%$ ige Stenose von 300 auf $230 \mathrm{~cm} / \mathrm{s}$ heruntergesetzt $[20,21]$. Auch in der Konsensuskon- ferenz amerikanischer Radiologen [22] wurde die PSV-Grenzgeschwindigkeit für eine $50 \%$ Stenose auf $125 \mathrm{~cm} / \mathrm{s}$ und für eine $70 \%$ ige Stenosen auf $230 \mathrm{~cm} / \mathrm{s}$ festgesetzt.

Ziel dieser Auswertung ist es, trotz der multifaktoriellen Genese, Ursachen für die Diskrepanz und breite Streuung in unterschiedlichen Vergleichsstudien auf dem Hintergrund methodenspezifischer Ursachen und Probleme zu evaluieren und therapieorientiert die Stenoseparameter zu beurteilen.

\section{Methodik}

Die Studien und Metaanalysen seit 1990 aus MEDLINE via PubMed zur sonographischen Stenosegraduierung von ICAStenosen wurden mittels der Schlagwörter: „carotid stenosis“, „stenosis grading ", , duplex ultrasound“, , , angiography“, "plaque formation“, „eccentric plaque“ aufgesucht und evaluiert, insbesondere auch eine jüngere Publikation, die als einzige nach den multiparametrischen Kriterien der DEGUM eine Bewertung der sonographischen Stenosegraduierung vornimmt. Beim Vergleich der Wertigkeit von Duplexsonographie mit anderen radiologischen Untersuchungs- 
Gefässchirurgie 2020 25:91-104 https://doi.org/10.1007/s00772-020-00616-5

(c) Der/die Autor(en) 2020

\section{W. Schäberle}

\section{Sonographische Graduierung von Karotisstenosen. Einflussgrößen auf die hämodynamische Stenosegraduierung}

\section{Zusammenfassung}

Hintergrund. Zur Validität der Ultraschalldiagnostik bei Karotisstenosen und in den Stenosekriterien gibt es erhebliche Diskrepanzen. Ziel der vorliegenden Arbeit ist die Aufarbeitung der differierenden Kriterien anhand der Studienlage.

Methodik. Berücksichtigt werden die in PubMed zum Thema "sonographische Graduierung von Stenosen der A. carotis interna (ICA)" publizierten Artikel von 1990 bis 2019. Suchworte: „, carotid stenosis", "stenosis grading ", , duplex ultrasound“, , angiography“, "plaque formation“, „eccentric plaque“. Ergebnisse. Bei der Graduierung von hochgradigen ICA-Stenosen zeigen ROCKurven in Vergleichsstudien zwischen Angiographie und Duplexsonographie (FKDS) mit Messung der intrastenotischen, systolischen Spitzengeschwindigkeit (PSV) zwar in der Diagnostik höhergradige Stenosen mit Sensitivitäten und Spezifitäten um $90 \%$ und eine gute Korrelation, jedoch eine bedeutende Streubreite einzelner PSVMesswerte.

Eine gravierende bisher in Studien wenig berücksichtigte Diskrepanz zwischen hämodynamischen (PSV) und morphologischen Messparametern (angiographische Diameterreduktion) verursacht die exzentrische Plaqueformation: Exzentrische Plaque erzeugen eine deutlich geringere Flächenreduktion und somit geringere hämodynamische Relevanz als konzentrische und somit auch eine deutlich geringere PSV (bei 50\% Stenose bis $30 \%$ Diskrepanz). Bei gleicher Diameterreduktion ist das Embolierisiko bei exzentrischen Plaques durch ihre größere Plaquedicke jedoch erhöht.

Schlussfolgerungen. Diskrepanzen in der Stenosegraduierung zwischen Angiographie und FKDS werden durch die Plaqueformation bzw. Plaquekonfiguration mitverursacht. Bei höhergradigen, exzentrischen Stenosen mit hoher Plaquedicke muss das Dilemma der unterschätzten Emboliegefahr bei durch hämodynamische Parameter graduierten Stenosen in der Therapieplanung mitbedacht werden.

\section{Schlüsselwörter}

A. carotis interna - Duplexsonographie . Angiographie - Stenosegraduierung · Plaqueformation

\section{Ultrasound grading of carotid artery stenosis. Factors affecting the hemodynamic stenosis grading}

\section{Abstract}

Background. There are considerable discrepancies regarding the validity of ultrasound diagnostics and the criteria for carotid artery stenosis. The aim of this publication is an evaluation of the varying criteria based on the study situation.

Methods. The articles published in PubMed on the topic ultrasound grading of stenosis of the internal carotid artery (ICA) from 1990 to 2019 were taken in account. Search items: carotid stenosis, stenosis grading, duplex ultrasound, angiography, plaque formation and eccentric plaque.

Results. While comparative studies of angiography and color duplex ultrasound (CDU) with measurement of intrastenotic peak systolic velocity (PSV) based on the analysis of receiver-operating characteristic (ROC) curves showed sensitivities and specificities around $90 \%$ and a good correlation, there was a considerable scatter of individual measurement parameters. Eccentric plaque formation is a major factor contributing to the discrepancy between hemodynamic (PSV) and morphological (angiographic diameter reduction) stenosis grading but is often ignored in studies. Eccentric plaques cause a markedly smaller area reduction with less hemodynamic relevance than concentric plaques, resulting in a markedly lower PSV (which may cause a discrepancy of up to $30 \%$ when $50 \%$ stenosis is present); however, eccentric plaques causing the same diameter reduction as concentric plaques are thicker and thus have a higher risk of embolism. Conclusion. Different plaque formations and configurations can lead to discrepancies in stenosis grading between angiography and CDU. When higher grade eccentric stenosis is caused by greater plaque thickness, treatment planning cannot be based on the hemodynamic stenosis grade alone but should also take the dilemma of an underestimated risk of embolism into account.

Keywords Internal carotid artery - Duplex ultrasound . Angiography · Stenosis grading · Plaque formation methoden wurde insbesondere nach Ursachen für die Diskrepanz in der Stenosegraduierung nach der PSV-Grenzgeschwindigkeit zwischen den Studien sowie nach Ursachen für die Streuung der Messwerte innerhalb der Studien gesucht. Dabei wurden methodische und hämodynamische Einflussfaktoren beleuchtet sowie eine mögliche Verbesserung durch Anwendung der multiparametrischen Stenosekriterien reflektiert. Die sonographische Messme- thodik, insbesondere auch die Hauptund Zusatzkriterien der DEGUM werden auf die Möglichkeit struktureller Fehler untersucht.

\section{Ergebnisse}

\section{Stenosegraduierung durch morphologische und hämo- dynamische Parameter im Vergleich}

Farbkodierte Duplexsonographie (FKDS), CTA und MRA zeigen vergleichbare Sensitivitäten und Spezifitäten von jeweils um $90 \%$ bei hochgradigen $(>70 \%)$ und somit eventuell 


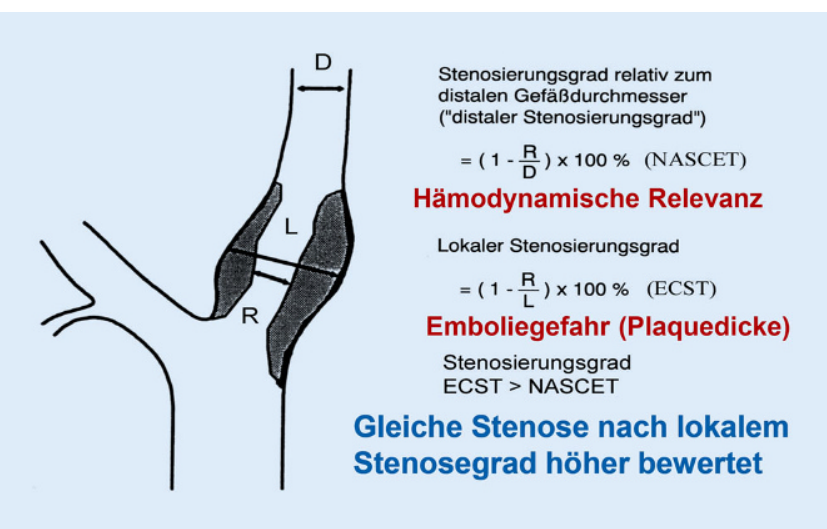

Abb. 1 A Schematische Darstellung der Stenosegraduierung nach dem distalen und nach dem lokalen Stenosegrad. Wenn es bei der Karotisstenose primär um die Durchblutungsminderung durch die Stenose ginge, wäre der distale Stenosegrad die adäquate Messmethode. Das Embolierisiko, das von der Stenose ausgeht und das von der Plaquedicke abhängt, wird jedoch im lokalen Stenosegrad besser abgebildet. ( $D$ distaler Karotisdurchmesser, $L$ Bulbusdurchmesser, $R$ Residuallumen)

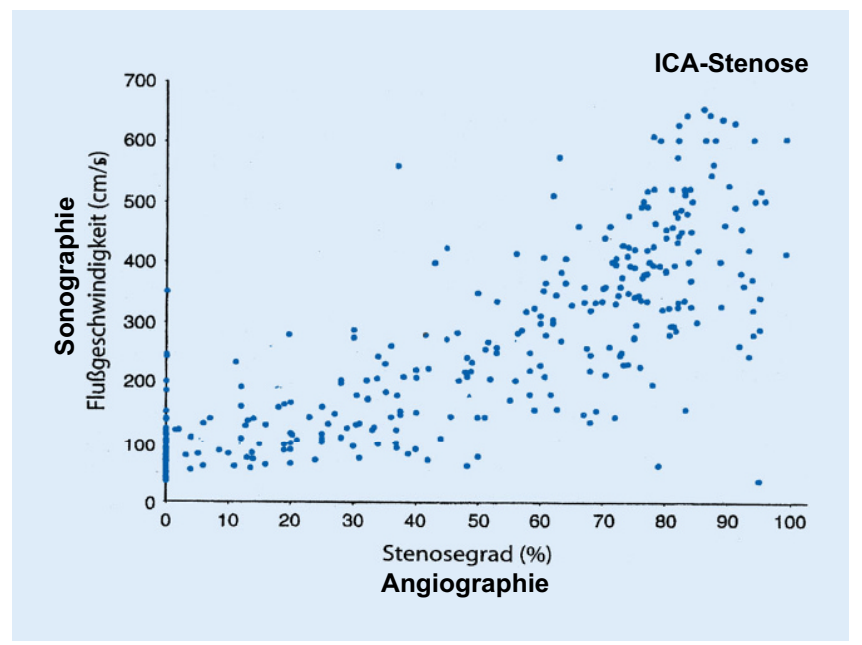

Abb. 2 A Karotisstenose: Der Vergleich zwischen dem angiographisch bestimmten und dem sonographisch über die PSV Erhöhung gemessenen Stenosegrad zeigt zwar eine gute Korrelation jedoch eine große Streubreite einzelner Wert. (Aus [2], mit freundlicher Genehmigung von Elsevier) interventionsbedürftigen ICA-Stenosen im Vergleich zur Angiographie: Sensitivität/Spezifität: FKDS $0,89 / 0,84$; MRA 0,88/0,84; CE-MRA 0,94/0,93, CTA 0,77/0,95. [23, 24]. Bei Stenosen von $50-69 \%$ zeigen die nichtinvasiven Methoden, insbesondere auch die FKDS im Vergleich zu Angiographie in einer Metaanalyse schlechte Sensitivitäten bei jedoch guten Spezifitäten. Sensitivität/Spezifität: FKDS $0,36 / 0,91$; MRA 0,37/0,91; CE-MRA 0,77/0,97; CTA 0,67/0,79 [17, 18]. Ein jüngst publiziertes systematisches Review (1990 bis 2018) zeigt bei $>70 \%$ igen ICA-Stenosen eine vergleichbar gute Sensitivität und Spezifität für CTA $(90,6 \% / 93 \%)$ und FKDS mit dem Stenosekriterium PSV (92,3\%/89\%). Unter Kombination der PSV mit der intrastenotischen, enddiastolischen Geschwindigkeit in der FKDS verbesserte sich die Sensitivität sogar auf 98,7\% und die Spezifität auf 94,1 \% [25]. Demgegenüber steht in einer multiparametrischen Analyse nach DEGUMUltraschallkriterien im Vergleich zur Angiographie [15] eine vergleichsweise schlechte Sensitivität der FKDS von $81 \%$ bei $>70 \%$ igen Stenosen und von nur $35 \%$ bei 50-69\%igen Stenosen (ROC Kurven).

\section{Multiparametrische Ultraschall- kriterien der DEGUM und strukturelle Fehlermöglichkeiten}

Trotz abgesenkten PSV-Grenzgeschwindigkeiten in angloamerikanischen Studien halten die DEGUM und die Deutsche Gesellschaft für Neurologie an den alten NASCET-Kriterien mit der relativ hohen Grenzgeschwindigkeit von $300 \mathrm{~cm} / \mathrm{s}$ für $>70 \%$ ige Stenosen fest, weil dadurch eine Vergleichbarkeit sowohl in Studien als auch in Patientenverlaufsuntersuchungen erreicht wird. Durch Anwendung der Zusatzkriterien soll die etwas hohe Grenzgeschwindigkeit korrigiert werden. Die Einflussfaktoren aufdie hämodynamische Graduierung müssen dabei jedoch berücksichtigt werden.

\section{PSV intra- und poststenotisch}

Resümierend muss aus der Geschichte der sonographischen Stenosegraduierung festgehalten werden, dass es trotz des Versuchs einer Vereinheitlichung der Methodik für die Stenosegraduierung keine international einheitliche PSVGrenzgeschwindigkeit für eine $>70 \%$ Stenose nach dem distalen Stenosegrad (NASCET Kriterium) gibt, sondern dass wir hier nach DEGUM mit $300 \mathrm{~cm} / \mathrm{s}$ [14] fast ein Drittel über den angloamerikanisch gebräuchlichen und in den Leitlinien der ESVS empfohlenen $230 \mathrm{~cm} / \mathrm{s}[12,22]$ liegen.
Der Vergleich zwischen angiographisch (Diameterreduktion) und duplexsonographisch (Hämodynamik/PSV) bestimmtem Stenosegrad zeigt zwar eine gute Korrelation [2], jedoch eine beachtenswerte Streuung einzelner Messwerte (- Abb.2). Der Einfluss von systemischen Faktoren (RR, Gefäßwandrigidität) und der Einfluss der Kollateralfunktion auf die PSV sollen durch Einsatz der multiparametrischen Stenosegraduierung der DEGUM reduziert werden (• Tab. 1).

Neben dem international anerkannten, wichtigsten Hauptkriterium, der PSV, sind B-Bild und die plaquebedingte Lumeneinengung in der FKDS bei gering- bis mittelgradigen Stenosen mit sonomorphologisch ausreichend guter Plaquedarstellbarkeit ein wichtiges Kriterium, weil die hämodynamischen Parameter noch keine zuverlässig differenzierbare Veränderung zum Normalbefund aufweisen [14]. Höchstgradige, $>95 \%$ ige Stenosen zeigen bedingt durch intrastenotische Reibungsverluste, insbesondere bei langstreckigen Stenosen, nicht immer die erwartete hohe PSV. Dann kann die distal an der Schädelbasis gemessene PSV zurate gezogen werden ( Tab. 1), die nach hochgradigen Stenosen abfällt (Druckabfall über der Stenose). 


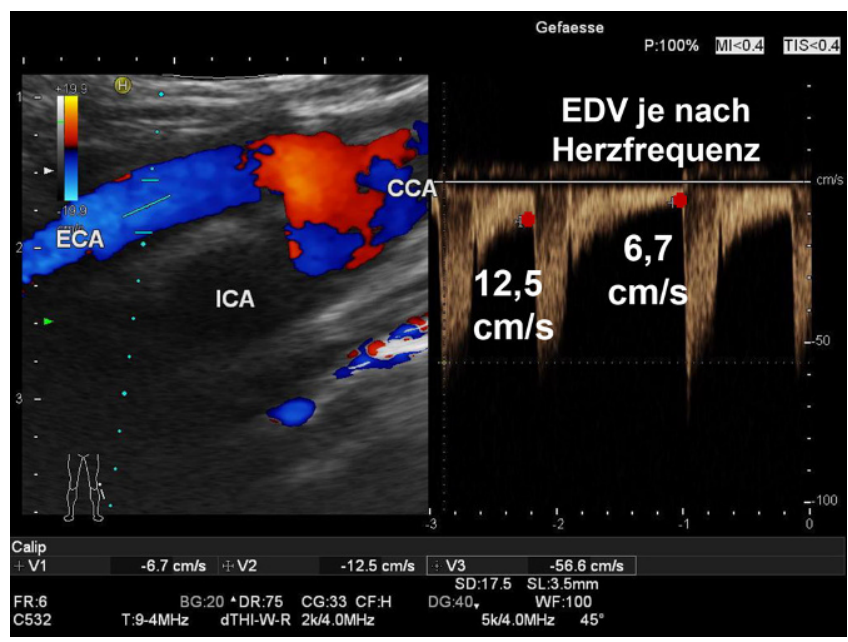

Abb. 3 ॥ Das Absinken der diastolischen Geschwindigkeit und somit der niedrigere Messwert in bradykarden Phasen wird bei einem Patienten mit absoluter Arrhythmie anschaulich dargestellt (in diesem Fall exemplarisch an der vergleichbaren ECA bei ICA-Verschluss). Bei zeitlich verkürzter Diastole, was einer Tachykardie entsprechen würde, ist der Messwert der enddiastolischen Geschwindigkeit in diesem Fall doppelt so hoch

\section{Beurteilung von Kollateralen (A. supratrochlearis)}

Ein weiteres Kriterium für das Vorliegen einer hochgradigen ICA-Stenose ist die Strömungsumkehr in als Kollateralen fungierenden Verbindungsarterien (intrakraniell - extrakraniell), wie in der sonographisch gut beurteilbaren Arteria supratrochlearis. Dieses Kriterium ist sehr spezifisch, jedoch weniger sensitiv, weil auch andere Kollateralkreisläufe (in unterschiedlich hohem Ausmaß) bei ICA-Stenosen einspringen können.

\section{Enddiastolische Geschwindigkeit (EDV)}

Die meisten übrigen Zusatzkriterien zeigen in der Stenosegraduierung eine schlechtere Korrelation [20] gegenüber der Angiographie als die PSV, meist bedingt durch andere wenig einschätzbare Einflussfaktoren auf diese Messgrößen.

Für eine $>70 \%$ Stenose zeigt eine EDV $>100 \mathrm{~cm} / \mathrm{s}$ eine Sensitivität von $82 \%$ und eine Spezifität von $90 \%$ [21]. Somit lässt sich durch die EDV als ergänzendes Stenosekriterium die Spezifität für die Diagnose einer $>70 \%$ Stenose verbessern, die EDV ist jedoch fehleranfällig, weil sie neben der Wandrigidität auch stark von der aktuellen Herzfrequenz abhängig ist (• Abb. 3). Während bradykarden Phasen sinkt die enddiastolische Phase längerstreckig ab (geringer enddiastolischer
Einfluss der Kollateralfunktion auf die intrastenotische PSV-Erhöhung

Die intrastenotische PSV wird einerseits von der Ausprägung der Kollateralen um das stenosierte Areal beeinflusst, andererseits davon, ob das Gefäß trotz Stenose selbst als Kollaterale mitwirkt. Die PSVErhöhung ist geprägt vom Druckabfall über der Stenose: Bei guter Kollateralisierung durch die kontralaterale ICA oder durch Vertebralarterien ist der Druckabfall über der Stenose geringer und eine etwas geringere PSV zu erwarten. Diese an peripheren Arterien bedeutungsvolle Beeinflussung der PSV durch Kollateralgefäße [26] ist in der Karotisstrombahn jedoch geringer.

Durch die Kollateralfunktion können bei hochgradiger Karotisstenose in der kontralateralen ICA höhere Flüsse und somit etwas erhöhte PSV-Werte auftreten. Dies kann bei bilateralen Karotisstenosen mit unterschiedlichen Stenosegraden zur Überschätzung der geringergradigen Stenose führen [27-29]. Nach Operation einer hochgradigen ICA-Stenose kann die kontralaterale ICA-Stenose eine geringere PSV im Vergleich zum präoperativ erhobenen PSV-Wert zeigen. Der präoperativ gemessene Wert täuscht kontralateral durch die Kollateralfunktion eine höhergradige Stenose vor. Die Beurteilung der A. carotis communis (CCA) und der PSV-Ratio kann jedoch Fehlinterpretationen durch die Kollateralfunktion korrigieren.

\section{Einfluss der exzentrischen Plaqueformation auf die hämodynamische Wirksamkeit und somit auf die PSV-Erhöhung}

Die Plaquekonfiguration bzw. Plaqueformation hat großen Einfluss auf die hämodynamische Wirksamkeit der Plaque und somit auf den PSV-Messwert. So zeigen konzentrische Plaque bei gleicher Diameterreduktion (angiographische Lumeneinengung) eine deutlich höhere hämodynamische Relevanz als exzentrische [28]: Eine konzentrische $50 \%$ ige Diameterreduktion entspricht einer 75\%igen Flächenreduktion (• Abb. 4) und führt $\mathrm{zu}$ einer PSV-Erhöhung von etwa $200 \mathrm{~cm} / \mathrm{s}$ nach dem lokalen Ste- 


\section{Konzentrischer} Plaque
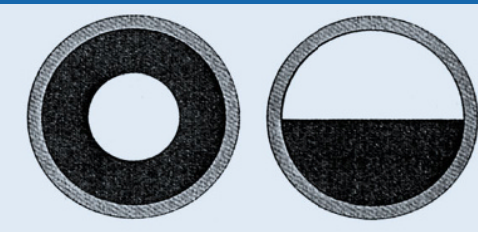

Morphologie

$50 \%$ Diameter

$50 \%$ Diameter $50 \%$ Fläche

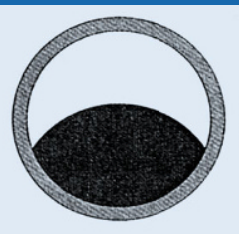

$50 \%$ Diameter $<50 \%$ Fläche

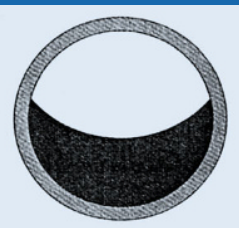

$50 \%$ Diameter $>50 \%$ Fläche aber $<75 \%$ Fläche

Hämodynamik

Fine

$\square=$ Lumen

PSV $200 \mathrm{~cm} / \mathrm{s}$ // PSV $120 \mathrm{~cm} / \mathrm{s}$ (nach lokalem Stenosegrad, ECST)

PSV $300 \mathrm{~cm} / \mathrm{s}$ // PSV $200 \mathrm{~cm} / \mathrm{s}$ (nach distalem Stenosegrad, NASCET)

Abb. 4 \ Hämodynamische Relevanz verschiedener Plaqueformen bei gleicher Diameterreduktion: Bei konzentrischer Stenose (links) entspricht eine Diameterreduktion um 50 \% einer 75\%igen Flächenreduktion. Das entspricht in der Karotisstenose einer PSV von $2 \mathrm{~m} / \mathrm{s}$ nach dem lokalen Stenosegrad und umgerechnet $3 \mathrm{~m} / \mathrm{s}$ nach dem distalen Stenosegrad. Bei gleicher Diameterreduktion führt eine exzentrische Stenose nur zu einer 50\%igen Flächenreduktion. Bei pilzförmigem Plaquewachstum ist die Flächenreduktion sogar noch geringer (vgl. dazu auch • Abb. 9). Daher ist bei diesen exzentrischen Stenosen bei gleicher Diameterreduktion durch die geringere hämodynamische Relevanz die PSV $120 \mathrm{~cm} / \mathrm{s}$ nach dem lokalen Stenosegrad und umgerechnet $200 \mathrm{~cm} / \mathrm{s}$ nach dem distalen Stenosegrad (nach von der DEGUM übernommenem NASCET-Kriterium). (Aus [28])

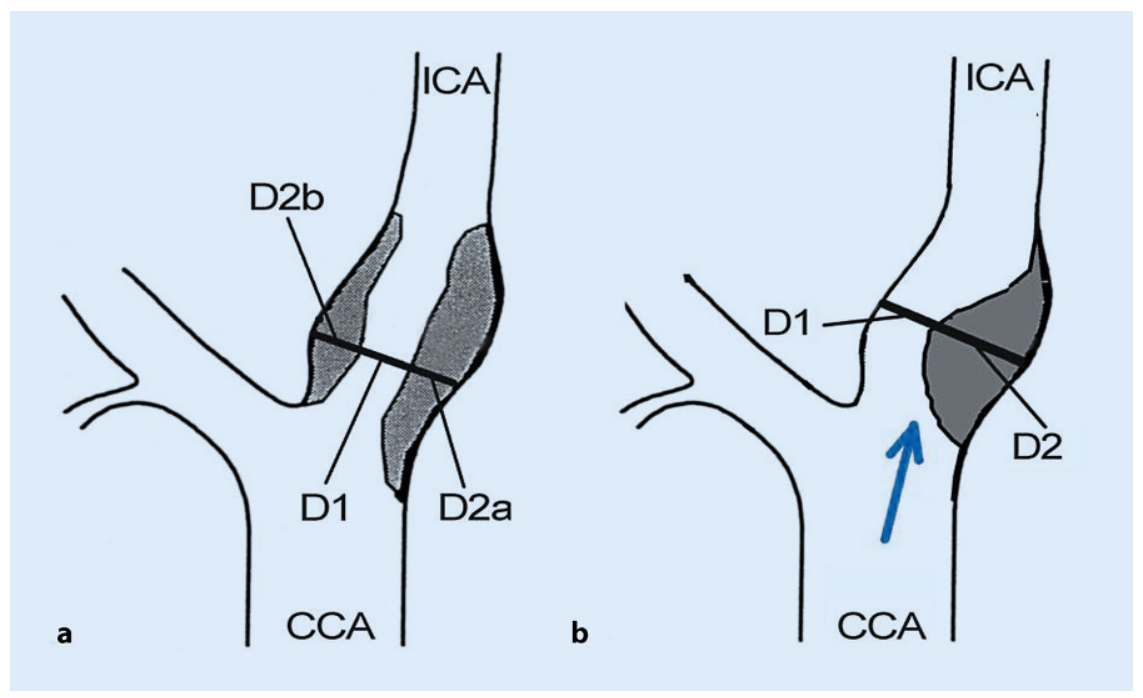

Abb. 5 ॥ Plaquekonfiguration bzw. Plaqueformation: Bei gleicher Diameterreduktion, also angiographisch gleichem Stenosegrad (im Beispiel. ca. $65 \%$ ige Internastenose nach lokalem Stenosegrad) zeigen exzentrische Lumeneinengungen (Bildabschnitt b: D1) trotz geringerer hämodynamischer Relevanz ein höheres Embolierisiko als konzentrische Stenosen (Bildabschnitt a: D1). Höhere Scherkräfte (blauer Pfeil) führen an einer exzentrischen Plaque bei größerer Plaquedicke (Bsp.: doppelte Plaquedicke: D2a + D2b = D2) zu einem höheren Embolierisiko

nosegrad sowie von $250-300 \mathrm{~cm} / \mathrm{s}$ nach dem distalen Stenosegrad. Demgegenüber verursacht eine exzentrische Plaque von 50\%iger Diameterreduktion (also gleicher Stenosegrad nach angiographischer Graduierung) nur eine 50\%ige Flächenreduktion und somit eine PSV von $150-200 \mathrm{~cm} / \mathrm{s}$ nach dem distalen Stenosegrad und von $120 \mathrm{~cm} / \mathrm{s}$ nach dem lokalen Stenosegrad. Exzentrische Plaque sind damit hämodynamisch viel weniger relevant und zeigen auch eine deutlich geringere PSV [28], sie haben jedoch bei gleicher Diameterreduktion eine deutlich höhere Plaquedicke. Durch die höhere Wirkung von Scherkräften ist das Embolierisiko erhöht (• Abb. 5 und 6).

\section{Messfehler}

Der gravierendste Messfehler entsteht durch Dopplerwinkeleinstellfehler. Weil die Strömungsvektoren normal parallel zur Gefäßwand verlaufen, wird die Winkelkorrektur an dieser ausgerichtet. Exzentrische Plaque führen jedoch zur Ablenkung der Strömungsvektoren $(\bullet$ Abb. $6 a, b)$, sodass die adäquate Winkelkorrektur anhand des farbduplexsonographischen, bei adäquater Geräteeinstellung am durch das Aliasing darstellbaren, intrastenotischen Strömungsjet ausgerichtet werden sollte [28]. Sonst entsteht durch die inadäquate Winkelkorrektur bei exzentrischer Plaqueformation ein weiterer gravierender PSV-Messfehler.

\section{》) Der gravierendste Messfehler entsteht durch Dopplerwinkeleinstellfehler}

Weiterhin können divergierende Strömungsvektoren im Stenosejet und in der turbulenten Strömung direkt nach der Plaque zu Winkeleinstellfehlern und zur Überbewertung der PSV führen [19]. Die Auswirkung eines oft unvermeidbaren Winkeleinstellfehlers auf die PSV ist jedoch umso geringer je spitzwinkliger der Dopplerwinkel ist (Kosinusfunktion). 

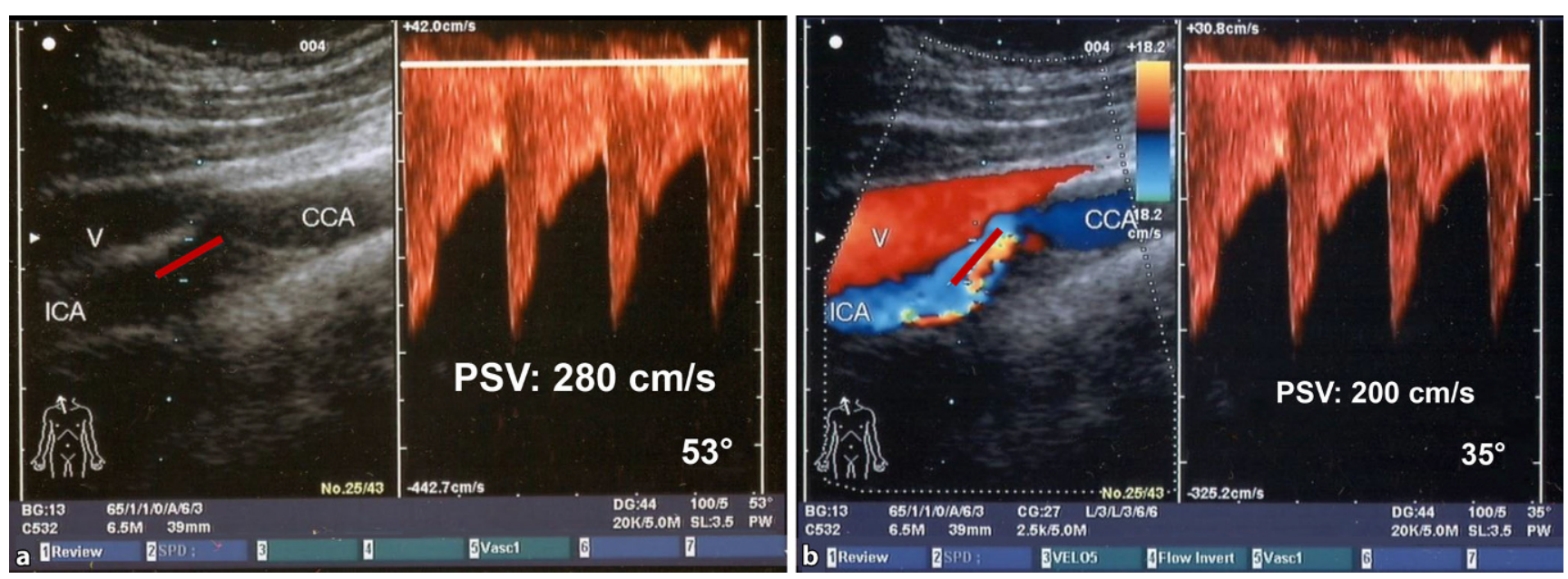

Abb. 6 ॥ Einfluss des Dopplerwinkels auf die PSV-Messung. a Wegen normalerweise parallel zur Gefäßwand verlaufenden Strömungsvektoren wird die Winkelkorrektur (rot markiert) des Dopplerwinkels folgerichtig parallel zur Gefäßwand eingestellt (Dopplerwinkel hier $53^{\circ}$ ). Die gemessene PSV von $280 \mathrm{~cm} / \mathrm{s}$ entspricht hier einer ca. 70\%igen Stenose (NASCET-Kriterium). b Bei gerätetechnisch adäquat eingestelltem Farbdopplerbild zeigt das Aliasing im Strömungsjet der Stenose, dass die Strömungsvektoren zur exzentrischen Plaque hin abgelenkt werden. Der Dopplerwinkel (rot markiert), der hier entsprechend des abgebildeten Strömungsjets eingestellt wurde, beträgt $35^{\circ}$ (gegenüber $53^{\circ}$ der Einstellung parallel zur Gefäßwand). Die PSV von $200 \mathrm{~cm} / \mathrm{s}$ bedeutet eine etwa $50 \%$ ige Stenose (nach von DEGUM übernommenen NASCET-Kriterien). (Aus [28])

\section{Genese und Häufigkeit exzentrischer Plaque}

In jeder Gefäßbifurkation kommt es strömungsphysikalisch in der Flussteilung zu Separationszonen an der zum Flussteiler abgewandten Seite ( $\mathbf{0}$ Abb. 7a). In diesen Separationszonen kommt es zu sehr langsamen Strömungsgeschwindigkeiten mit zum Teil retrograden Strömungsanteilen. Dieser Effekt wird durch die Gefäßerweiterung im Bereich des Bulbus noch verstärkt. Die Strömungsverlangsamung in Separationszonen erzeugt eine turbulente Strömung, mit zum Teil senkrecht zur Gefäßwand gerichteten Strömungsvektoren und mit einem erhöhten Druck auf die Gefäßwand, wodurch eine Intimaschädigung erzeugt werden kann. Dies führt zur Plaquebildung ( $\bullet \mathbf{A b b}$. $7 \mathbf{b}$ ) von meist exzentrischer Plaque im Bulbus, vor allem an der zum Externaabgang gegenüberliegenden Gefäßwand [28]. Dazu wurde in einer eigenen Untersuchung bei 68 Patient(inn)en (Alter 51 bis begrenzt auf unter 75 Jahre; männlich 47, weiblich 21) mit einer Karotisstenose zwischen 30 und $50 \%$ (von DEGUM übernommenes NASCET-Kriterium: PSV $120-200 \mathrm{~cm} / \mathrm{s}$ ) die Plaqueformation evaluiert. In $79 \%$ der Fälle war die Stenose durch exzentrische Plaques bedingt, die in $88 \%$ an der zum Externaabgang gegenüberliegenden Bulbuswand, also in der Separationszone lokalisiert waren (• Abb. 6c). Nach Weiterentwicklung zu hochgradigen Stenosen und bei älteren Patienten ist bedingt durch sekundäre Turbulenzen und andere Einflussfaktoren auf die Arteriosklerose dieser Effekt einer primären Plaquegenese nicht mehr derart eindrucksvoll nachweisbar. Nur eine Studie [30] verweist auf den hohen Anteil von exzentrischen Plaques mit $74 \%$ gegenüber überwiegend konzentrischen Plaques in $26 \%$ bei $>50 \%$ Stenosen (NASCET-Kriterium). Postmortem-Studien bestätigen die Häufigkeit von exzentrischen Plaques in der Separationszone des Bulbus [31, 32].

\section{Diskussion}

In dieser methodenkritischen Evaluation wurden speziell die Vergleichsstudien mit anderen bildgebenden Verfahren, insbesondere der Angiographie, ausgewertet, die zur Erklärung und Interpretation der methodenspezifischen Probleme und Diskrepanzen beitragen. Vergleiche mit MRA und CTA wurden weniger berücksichtigt, weil die Diskussion der methodenspezifischen Probleme dieser Untersuchungsmethoden den Rahmen dieser Auswertung sprengen würde.
Metaanalysen zeigen, dass zur adäquaten Stenosegraduierung bei höhergradigen und somit eventuell rekonstruktionsbedürftigen ICA-Stenosen die FKDS mit Sensitivitäten und Spezifitäten von ca. $90 \%$ zeigt und deshalb keine weitere radiologische Kontrollbildgebung benötigt wird. Bei Stenosen $<70 \%$ zeigt die FKDS jedoch schlechtere Sensitivitäten [23-25].

\section{》) Bei Stenosen $<70 \%$ zeigt die FKDS schlechtere Sensitivitäten}

Die Stenosekriterien sind jedoch nicht einheitlich [13]. Der verlässlichste, am besten quantifizierbare Wert in der sonographischen Stenosegraduierung der Karotisstenose ist die intrastenotisch gemessene systolische Spitzengeschwindigkeit (PSV). Diese zeigt auch die höchste Korrelation zur Angiographie mit 0,83 bei 70 bis $99 \%$ Stenosen gegenüber einer Korrelation von 0,75 für die enddiastolische intrastenotische Geschwindigkeit und 0,6 für die PSV-Ratio (ICA/CCA) [20]. Auffallend und auch verunsichernd ist die diskrepante Grenzgeschwindigkeit verschiedener Studien innerhalb des distalen Stenosegrads (NASCET-Kriterium) von $325 \mathrm{~cm} / \mathrm{s}$ für $>70 \%$ ige Stenosen (ursprüngliches NASCET-Kriterium) 

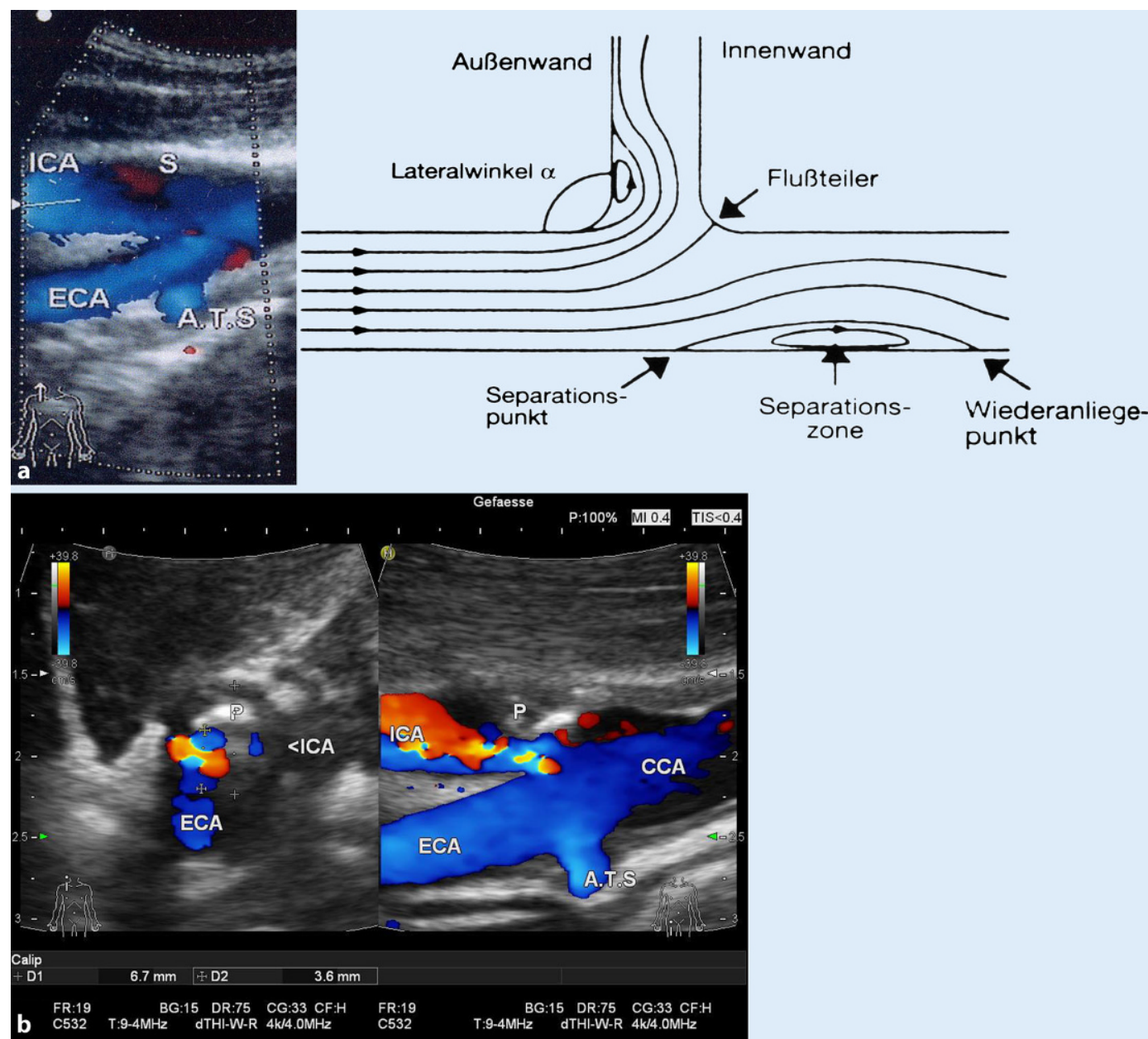

Abb. 7 A Plaquegenese. a In Gefäßbifurkationen kommt es durch Ablenkung der Strömungsvektoren zu Separationszonen an der zur Flussteilung gegenüberliegenden Gefäßwand (rechter Bildabschnitt). Die deutlich verlangsamte, zum Teil retrograd zirkulierende Strömungsgeschwindigkeit führt zu Turbulenzen mit Strömungsvektoren, die zum Teil senkrecht zur Wand gerichtet sind und zu einer reduzierten Schubspannung mit lokal erhöhtem Druck auf die Gefäßwand führen, wodurch eine Intimaschädigung verursacht werden kann. Die Separationszonen (S) lassen sich auch farbduplexsonographisch (linker Bildabschnitt) im gesunden Bulbus an einem Farbumschlag (von blau nach rot) nahe der Gefäßwand gegenüber des Externaabgangs (ECA) zeigen (A.T.S: A. thyroidea superior). $\mathbf{b}$ Häufige Lokalisation der Plaquebildung ( $P$ ) in der Separationszone des Bulbus (ICA) an der Gefäßwand gegenüber des Externaabgangs (ECA)

[16] bis $230 \mathrm{~cm} / \mathrm{s}$ in der Konsensuskonferenz amerikanischer Radiologen [22].

In einer deutschen Konsensuskonferenz [14] wurden die ECST-Kriterien in die NASCET-Kriterien umgesetzt und dabei die relativ hohe systolische Grenzgeschwindigkeit von $300 \mathrm{~cm} / \mathrm{s}$ für eine 70 \%ige Stenose gewählt. Oberstes Ziel war es dabei, die durch die verschiedenen Stenosekriterien (lokaler versus distaler Stenosegrad) entstandene Unsicherheit sowie die dabei entstandenen unterschiedlichen Stenosegrade zu beseitigen und insbesondere auch in Verlaufskontrollen vergleichbare Werte zu erhalten, um eine Stenosegradzunahme adäquat einzuschätzen zu können sowie in Studien vergleichbare Verhältnisse zu schaffen.

Die aktuellen ESVS-Guidelines [12] empfehlen die FKDS als primäre Diagnostik in der Stenosegraduierung, verwenden jedoch das nach der amerikanischen radiologischen Konsensuskonferenz [22] revidierte NASCET-Kriterium als Grundlage (PSV $>230 \mathrm{~cm} / \mathrm{s}$ für eine Stenose $>70 \%$ und $>125 \mathrm{~cm} / \mathrm{s}$ für eine Stenose $>50 \%$ ). Bei derart unterschiedlicher Bewertung müsste eigentlich immer angegeben werden, nach welchem Kriterium die Stenose graduiert wird: nach DEGUM-NASCET-Richtlinien, der ESVSNASCET-Empfehlung oder nach dem
ECST-Kriterium, das immer noch den Vorteil hat, dass es die Plaquedicke besser berücksichtigt. Aufdiesen Vorteil verweisen auch die ESVS-Leitlinien: Eine Stenose durch eine voluminöse Plaque in einem dilatierten Bulbus wird im lokalen Stenosegrad (ECST-Kriterium) besser eingeschätzt [12], wodurch bei exzentrischen Plaques die Emboliegefahr besser erfasst wird ( $\triangle$ Abb. 1 und 5 rechter Abschnitt).

Aufgrund der Diskrepanzen wäre es in Deutschland sinnvoll, in einer erneuten Konsensuskonferenz eine therapieorientierte Stenosegraduierung nochmals zu überdenken und zumindest eine Vereinheitlichung der Grenzgeschwindigkeiten 
nach dem NASCET-Kriterium anzustreben. Die PSV-Werte nach dem revidierten NASCET-Kriterium liegen näher am ECST-Kriterium als am ursprünglichen NASCET Kriterium (•Tab. 1).

Einerseits ist es problematisch, dass in der amerikanischen [22] und der deutschen Konsensuskonferenz [14] trotz zugrunde liegendem NASCET-Kriterium unterschiedliche PSV-Werte als Grenzgeschwindigkeit für $>70 \%$ ige Karotisstenosen gewählt wurden (PSV $230 \mathrm{~cm} / \mathrm{s}$ versus $300 \mathrm{~cm} / \mathrm{s}$ ). Überraschenderweise ergeben andererseits die ROC-Kurven einer Studie [20] in diesem PSV-Bereich keine derart gravierenden Unterschiede in Sensitivität und Spezifität. So zeigt eine Senkung der PSV von 280 auf $230 \mathrm{~cm} / \mathrm{s}$ einen Anstieg der Sensitivität von 95 auf $99 \%$ und einen Anstieg des NPV von 89 auf $97 \%$, die Spezifität fällt jedoch von 93 auf $86 \%$ ab und der PPV von 97 auf $93 \%$. Die Treffsicherheit zeigt zwischen 94 und $95 \%$ kaum Veränderung [20].

Bei all den auf ROC-Kurven basierenden Zahlenspielen ist für die Zielsetzung der adäquaten Stenosegraduierung mit $\mathrm{zu}$ beachten, ob eher eine höhere Sensitivität oder eine höhere Spezifität erzielt werden soll. Bei der Festlegung auf eine Grenzgeschwindigkeit nach ROCKurven bedeutet die Wahl einer geringeren PSV als Grenzgeschwindigkeit eine höhere Sensitivität und eine höhere NPV. Sie führt jedoch zu einer geringeren Spezifität und einer geringeren PPV. Um bei geringerer PSV als Grenzgeschwindigkeit die Spezifität zu erhöhen, wird angloamerikanisch manchmal die PSVRatio als ergänzendes Kriterium hinzugenommen [20-22].

Die bei dem relativ hohem PSVWert der deutschen Konsensuskonferenz zwangsläufig etwas geringere Sensitivität soll durch die zusätzlichen Hauptund Zusatzkriterien in der multiparametrischen Stenoseanalyse (• Tab. 1) korrigiert werden.

Untermauert wird die Methode einer multiparametrischen Stenosegraduierung durch die Erfahrung aus älteren Vergleichsstudien zwischen dopplersonographischen akustischen Untersuchungen und der Angiographie gegenüber der pathologischen Aufarbeitung des Thrombendarteriektomiepräparats

als eine Art „golden standard“ [33-35]. Die ebenfalls multiparametrisch durchgeführte akustische Dopplersonographie (intrastenotische Dopplerfrequenzanalyse und -erhöhung, Analyse der Turbulenzen, Flussreduktion in der CCA und der ICA distal der Stenose und Flussanalyse der transorbitalen Arterien) zeigt sogar eine Sensitivität von $95 \%$ und eine PPV von $96 \%$ bei $>70 \%$ ICA-Stenosen. Demgegenüber zeigt die Angiographie nur eine Sensitivität von $88 \%$ und eine PPV $92 \%$. [33]. Bei $<70 \%$ Stenosen ist die akustische Dopplersonographie weniger zuverlässig. Verwiesen wird aber auch auf die notwendige, hohe Untersuchererfahrung in der Bewertung der semiquantitativen Untersuchungsparameter. Die separat mit ausgewertete PSV (FKDS) zeigt singulär allerdings nur eine Sensitivität von $89 \%$ [33] und eine PPV von $93 \%$ (>70\% Stenose). Ein Vergleich mit dem Endarteriektomiepräparat zur Stenosegraduierung kann nur nach dem damals in Deutschland üblichen lokalen Stenosegrad (ECST-Kriterium) zuverlässig durchgeführt werden.

\section{》) Die geringere Sensitivität soll in der Stenosegraduierung durch zusätzliche Kriterien korrigiert werden}

Ergänzend zur intrastenotisch gemessenen PSV gelten in der multiparametrischen Analyse der DEGUM die distal (an der Schädelbasis) gemessene, vom Stenosegrad abhängige, reduzierte PSV und der retrograde Flussnachweis in der kollateralisierenden A. supratrochlearis als am besten quantifizierbare Kriterien. Beide Kriterien zeigen eine hohe Spezifität, jedoch durch unterschiedliche Kollateralisierungswege eine limitierte Sensitivität. Nicht festgelegt ist jedoch eine Hierarchisierung der Kriterien bei eventuell widersprüchlichen Befunden in der Stenosegraduierung innerhalb der Hauptoder Zusatzkriterien der multiparametrischen Analyse.

Dass die als Zusatzkriterium weiterhin angeführte intrastenotische, enddiastolische Geschwindigkeit von der Wandrigidität und auch von der Herzfrequenz abhängt (• Abb. 3), wird in der Literatur nicht diskutiert, ebenso wenig die Beeinflussung der PSV-Ratio durch die Hämodynamik der A. carotis externa [28].

Unter Berücksichtigung der multiparametrischen Kriterien der DEGUM wurde die FKDS (hämodynamische Parameter) bisher nur in einer einzigen, jüngst publizierten Untersuchung gegenüber morphologischen Verfahren wie der Angiographie evaluiert [15]. Die dabei gewählte Angiographie in 2 Ebenen kann aus methodischen Limitierungen inzwischen jedoch schwerlich Referenzmethode und "golden standard" sein und somit nicht zur Infragestellung der duplexsonographischen Stenosegraduierung herangezogen werden $[19,36]$. Je nach Schnittebene kommt es vor allem bei exzentrischer Plaque zur Überbewertung der hämodynamischen Relevanz. Wenn die Plaque nicht adäquat getroffen ist, ist auch eine Unterschätzung des Stenosegrads möglich. Zur möglichst adäquaten Stenosegraduierung ist zumindest ein Herausdrehen der Karotisstenose in 3 Ebenen nötig.

Weiterhin zeigen exemplarische Abbildungen dieser Studie [15] methodische bedingte Fehler in der Winkeleinstellung (vgl. - Abb. 6) und in der Messungenauigkeit durch Verwendung von Messprogrammen (ohne eine weitere genaue Beschreibung der Messmethodik) sowie Diskrepanzen durch die fehlende Berücksichtigung der Plaqueformation (vgl. - Abb. 2), die zur Aussage führen, dass in der Differenzierung von operationsrelevanten Befunden in fast $10 \%$ Fehleinschätzungen bestehen würden und die duplexsonographische Stenosegraduierung vor Intervention eine weitere Bildgebung wie die Angiographie zur Bestätigung erfordern würde. Die Sensitivität von vergleichsweise nur $81 \%$ bei $>70 \%$ Stenosen und die schlechte Sensitivität von $35 \%$ bei mittelgradigen Stenosen (50-69\%) sind jedoch möglicherweise in der Plaqueformation begründet. Die Spezifität betrug 69\% und $89 \%$.

Die aktuellen ESVS-Guidelines empfehlen bei operationsrelevanten Stenosebefunden nicht die routinemäßige Kontrolle durch eine Angiographie, sondern durch eine zweite qualifizierte 


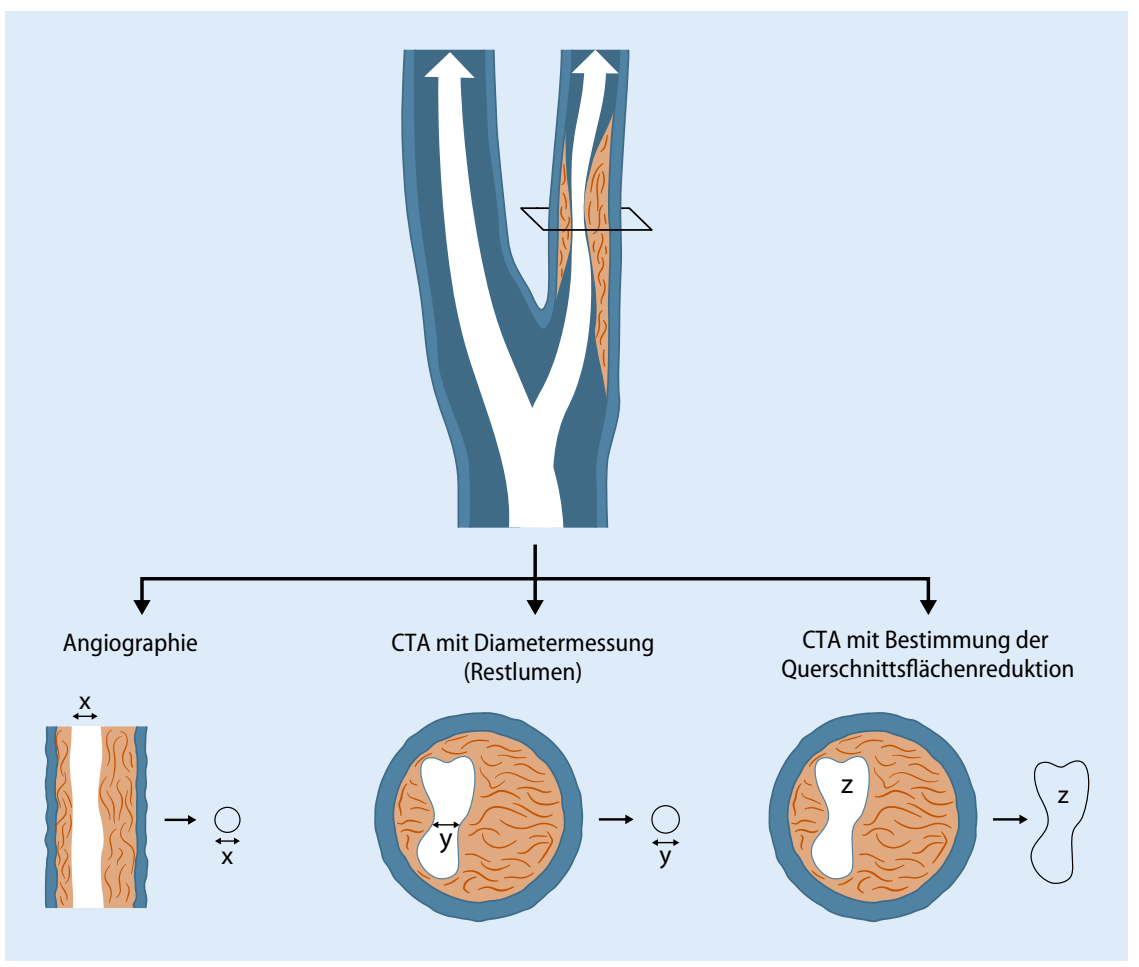

Abb. 8 ॥ Diameter-versus Flächenreduktion. Grafische Darstellung zum Vergleich von Angiographie (links), CTA mit Messung der Diameterreduktion (Bildmitte) und CTA mit Messung der Querschnittsflächenreduktion (rechts). Sie verdeutlicht die unterschiedlichen Stenosegradergebnisse zwischen Diameter- und Querschnittsflächenreduktion. Die duplexsonographische, hämodynamische Stenosegraduierung über die PSV basiert nach dem Kontinuitätsgesetz auf der Querschnittsflächenreduktion

Ultraschalluntersuchung oder die Bestätigung durch eine CTA oder MRA [12].

Bei allen Stenosegraduierungsparametern, die auf Hämodynamik basieren (alle Parameter mit Geschwindigkeitsmessungen) ist $\mathrm{zu}$ beachten, dass die Plaquekonfiguration einen entscheidenden Einfluss auf die hämodynamische Wirksamkeit der Stenose ausübt (• Abb.4). Konzentrische Plaque zeigen hämodynamisch eine deutlich ausgeprägtere Flächenreduktion. Die Stenosegraduierung über die PSV spiegelt so die hämodynamische Relevanz bzw. die Durchblutungsreduktion adäquater wider als die rein morphologischen Messungen der Diameterreduktion (Angiographie). Die schlechte Übereinstimmung der Duplexsonographie und der Angiographie bei mittelgradigen, häufig exzentrischen Stenosen kann somit durch die Anwendung der multiparametrischen Kriterien der DEGUM kaum verbessert werden.
Die Erweiterung der PSV durch die ergänzenden Zusatzkriterien der DEGUM kann auch keine zusätzliche Entscheidungshilfe zur Bewertung der Emboliegefahr durch die Plaqueformation bzw. Plaquekonfiguration liefern (• Abb. 5). Sie können nur innerhalb der auf Hämodynamik beruhenden Stenosegraduierung die Durchblutungsreduktion exakter quantifizieren und die komplexe hämodynamische Veränderung durch die Stenose besser beschreiben. Zur Emboliegefahr einer Stenose kann nur insofern Stellung genommen werden, als dass diese mit Zunahme des Stenosegrads also mit zunehmender Plaquedicke ansteigt $[17,18,37,38]$.

\section{》) Die Plaquekonfiguration beeinflusst die hämodynamische Wirksamkeit der Stenose}

Der Einfluss der Plaqueformation auf den duplexsonographisch gemessenen Stenosegrad (• Abb. 4) wird in Ver- gleichsstudien bisher kaum erwähnt und nicht berücksichtigt, obwohl dadurch die Streuung der Messwerte ( $\bullet$ Abb. 2) bei insgesamt guter Korrelation im Vergleich von Sonographie und Angiographie erklärt werden kann.

Die geringere Sensitivität der FKDS gegenüber der Angiographie [23] bei 50-69\%igen ICA-Stenosen kann durch die beschriebene Stenoseentstehung mit einem hohen Anteil an exzentrische Plaque in der Separationszone (vgl. - Abb. 6) verursacht sein [28, 30-32], weil exzentrische Plaque durch die geringere hämodynamische Relevanz eine geringere PSV zeigen (vgl. - Abb. 4). Von der Angiographie werden diese häufigen exzentrischen Stenosen durch die Wahl der zweidimensionalen Projektionsebenen mit dem per definitionem festgelegten, höchsten Stenosemaß in einer Ebene im Vergleich zur sonographisch gemessenen, hämodynamischen Relevanz systematisch überschätzt [28], wodurch im Methodenvergleich für die Sonographie geringere Sensitivitäten entstehen. Hochgradige Stenosen entwickeln im Verlauf oft komplexere Plaqueformen mit oft konzentrischeren Anteilen. Diese führen dann zu einer besseren Vergleichbarkeit von hämodynamischen und morphologischen Parametern und somit zu besseren Sensitivitäten. Diskrepanzen in der Stenosegraduierung zwischen Angiographie (Diameterreduktion) und FKDS (nach dem Kontinuitätsgesetz Ausdruck der Querschnittsflächenreduktion) sind also methodenspezifisch begründet und insbesondere bei exzentrischen Plaques unausweichlich (• Abb. 8).

Eine Vergleichsstudie zwischen der CTA und der FKDS, wobei die FKDS als Referenzmethode genommen wurde, bestätigt auch, dass die in der CTA bestimmte Flächenreduktion der duplexsonographischen, hämodynamischen Stenosegraduierung mit guter Korrelation sehr zuverlässig entspricht. Die mit der Angiographie vergleichbare Messung der Diameterreduktion in der CTA zeigt eine schlechtere Übereinstimmung mit der Graduierung über die PSV. [39]. Weiterhin zeigt auch ein CTA-interner Vergleich zwischen Diameterreduktion und Flächenreduktion im Vergleich zur 

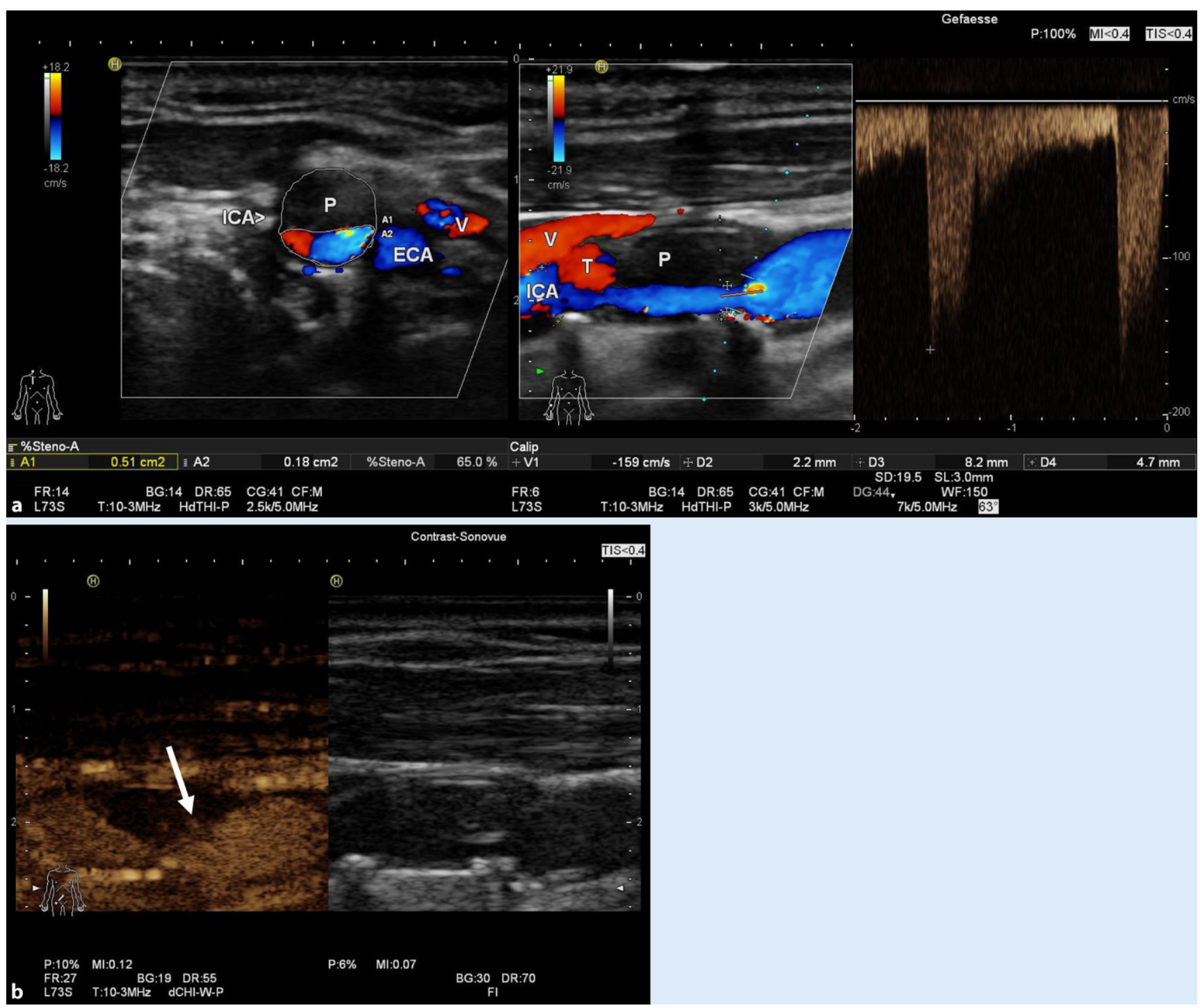

Abb. 9 ム Risikobewertung exzentrischer Stenosen. a Ausgeprägte exzentrische Stenose (gegenüber des Externaabgangs) mit einer Plaquedicke von fast $5 \mathrm{~mm}$, die im seitlichen Bild als hochgradige Stenose imponiert (entsprechend Angiographie), jedoch mit einer PSV von $160 \mathrm{~cm} / \mathrm{s}$ hämodynamisch nur einer mittelgradigen Stenose entspricht (vgl. Videoclip 1: im Plaqueverlauf zeigt sich nirgends ein PSV-Messwert von $>160 \mathrm{~cm} / \mathrm{s}$ ). Das Querschnittsbild (gut abgrenzbare Plaqueoberfläche farbduplexsonographisch gut darstellbar) entspricht dann planimetrisch einem lokalen Stenosegrad von $60 \%$ (dies würde umgerechnet einem distalen Stenosegrad von 30-40\% entsprechen). Bei diesem Patienten im Stadium I besteht nach hämodynamischen Parametern der Stenosegraduierung keine Operationsindikation. b Kontrastmittelsonographisch (CEUS) zeigen sich jedoch bei diesem Pateinten ein ausgeprägter exzentrischer Plaque (Plaquedicke $>4 \mathrm{~mm}$ ) und an der vorderen Plaquekante ein kleines Plaqueulkus, bedingt durch an der Plaquevorderkante ansetzende Scherkräfte (vgl. Videoclip 2)

Angiographie eine deutlich schlechtere Korrelation bei exzentrischen Stenosen als bei konzentrischen Stenosen [39]. Ein weiterer Vergleich von Diameterreduktion und Querschnittsflächenreduktion in einer CTA zur Stenosegraduierung [40] beschreibt auch eine ähnlich gute Korrelation $(0,93)$ wie der Vergleich von FKDS und Angiographie (Diameterreduktion), jedoch eine ähnliche Streuung der Messwerte mit hoher Abweichung in einzelnen Fällen. Diese Auswertun- gen bestätigen die Zuverlässigkeit der duplexsonographischen Stenosegraduierung hinsichtlich der Flächenreduktion und somit der hämodynamischen Relevanz. Die Querschnittsflächenreduktion in der CTA entspricht nach dem Kontinuitätsgesetz der vom Stenosegrad abhängigen PSV-Erhöhung in der FKDS [28].

Vorteile der CTA gegenüber der Angiographie als Referenzmethode zur FKDS sind neben der geringeren In- vasivität, die bessere Visualisierbarkeit von Plaquekonfiguration und -morphologie sowie die multiplanare Rekonstruktionsmöglichkeit, die eine genauere Stenosegraduierung über die Querschnittsflächenreduktion erlaubt und der duplexsonographischen Stenosegraduierung nach dem Kontinuitätsgesetz eher entspricht. Die Beurteilung von Plaquedicke und -charakter sowie Stenosegrad führt im Querschnitt der CTA auch zur treffsicheren Graduierung über 
den von der DEGUM früher favorisierten lokalen Stenosegrad.

Exzentrische Plaque sind gegenüber konzentrischen jedoch emboliegefährlicher, weil sie bezogen auf die gleiche Diameterreduktion lokal eine höhere stenosierende Plaquedicke haben (vgl. - Abb. 5; [28]). So wurden zerebrovaskuläre Ereignisse bei lokal $>70 \%$ ICAStenosen $(>50 \%$ nach NASCET) durch exzentrische Stenosen (höhere Plaquedicke) in $13,5 \%$ beschrieben gegenüber $5,5 \%$ bei konzentrischen Stenosen [30], obwohl die Kontaktfläche des Blutstroms bei konzentrischen Plaques höher ist als bei exzentrischen Plaques. In dieser Studie [30] hat die Plaqueform (exzentrisch) sogar einen deutlich stärkeren Einfluss auf die Plaquevulnerabilität als die sonographische Plaquemorphologie (Plaqueechogenität, Plaqueirregularität). Intimaverdickung und Plaque entstehen vor allem in Wandregionen mit wenig Schubspannung, Turbulenzen und Strömungsvektoren zum Teil senkrecht zur Gefäßwand, wie in der Separationszone des Bulbus. Simulationsmodelle zeigen, dass unterschiedliche Stärke und Verteilung der Schubspannung auf die Gefäßwand zwischen exzentrischen und konzentrischen Plaque eine entscheidende Rolle auf die Vulnerabilität spielen [41-44]. Die poststenotische Rezirkulationszone scheint zusätzlich einen Einfluss auf Thrombozytenauflagerungen und Thrombusformationen mit embolischem Risiko zu haben [45].

\section{》) Exzentrische Plaque sind gegenüber konzentrischen emboliegefährlicher}

Das Dilemma der hämodynamischen (duplexsonographischen) Stenosegraduierung der Karotis ist, dass diese häufigeren, exzentrischen Plaques mit höherer Plaquedicke bei gleichem angiographischem Stenosegrad (bisherige Grundlage für die statistische Risikobewertung zerebrovaskulärer Ereignisse) durch die geringere Flächenreduktion im Vergleich zu konzentrischen Plaque einen geringeren PSV-Wert und somit einen geringeren Stenosegrad zeigen (- Abb. 4 und 9a). Dies wird auch durch weitere hämodynamische Parameter in den Zusatzkriterien der multiparametrischen Analyse der DEGUM nicht korrigiert.

Die Plaquekonfiguration (exzentrisch, konzentrisch) lässt sich B-Bildsonographisch und in Kombination mit der FKDS gut beurteilen (• Abb. 9a), im Zweifel erlaubt eine ergänzend durchgeführte, kontrastmittelunterstützte Sonographie (CEUS) eine exzellente Aussage über die Plaqueform. (• Abb. 9b). So kann bei grauwertsonographisch höhergradiger, exzentrischer Lumeneinengung, jedoch ohne PSV-Erhöhung über $230 \mathrm{~cm} / \mathrm{s}$ (hämodynamisch $<70 \%$ Stenose), im Zweifelsfall durch die ergänzende kontrastmittelsonographische Untersuchung die Operationsindikation im Stadium I einer Karotisstenose abgewogen werden und durch die Mitbeurteilung der Oberfläche können schon durch Scherkräfte verursachte, ulkusbedingte Aussparungen entdeckt werden (Videoclip 1 und 2).

\section{1) Plaquekonfiguration muss zur Risikoabschätzung mit bewertet werden}

Für die Abschätzung der Emboliegefahr einer Karotisstenose ist allein die Bestimmung der hämodynamischen Relevanz, das heißt der Durchblutungsreduktion nicht ausreichend, die Plaquekonfiguration muss zur Risikoabschätzung mit bewertet werden. Unabhängig davon zeigen weitere Studien [46-48], dass Plaquemorphologie, (Plaqueoberfläche und Plaqueechogenität) sowie die in der kontrastmittelverstärkten Sonographie darstellbare Plaquevaskularisierung einen relevanten Einfluss auf die Emboliegefahr haben.

\section{Fazit für die Praxis}

- Die FKDS zeigt bei höhergradigen ICA-Stenosen im Vergleich zur Angiographie gute Sensitivitäten und Spezifitäten von etwa $90 \%$, vergleichbar mit anderen nichtinvasiven, jedoch teureren und belastenderen Methoden (CTA, MRA).
- Durch eine deutsche Konsensuskonferenz wurde die Stenosegraduierung vom ECST-Kriterium auf das NASCET-Kriterium umgestellt. Die dabei nach NASCET gewählte Grenzgeschwindigkeit von $300 \mathrm{~cm} / \mathrm{s}$ für eine $70 \%$ Stenose (ursprüngliches NASCET-Kriterium) wurde parallel dazu nach weiteren angloamerikanischen Studien auf $230 \mathrm{~cm} / \mathrm{s}$ nach unten korrigiert. Diese wurde auch in den ESVS-Leitlinien übernommen.

- In der Stenosegraduierung sollte eine Vereinheitlichung für das Hauptkriterium PSV zwischen DEGUM - NASCET und ESVS - NASCET angestrebt werden.

- Die im Bulbus häufig vorkommende exzentrische Plaquekonfiguration verursacht Diskrepanzen in der Stenosegraduierung zwischen Angiographie und FKDS.

- Die Entwicklung von exzentrischen Plaques im Bulbus wird in der Separationszone des Flussteilers durch reduzierte Schubspannung, turbulente Strömung und erhöhten Wanddruck begünstigt.

- Die PSV drückt die hämodynamische Wirksamkeit einer Stenose nach dem Kontinuitätsgesetz gut aus. Die Einflussgrößen auf die PSV müssen berücksichtigt werden, um die Emboliegefahr nicht zu unterschätzen, wenn bei exzentrischen Plaques eine geringere PSV einen geringeren hämodynamischen Stenosegrad (Flächenreduktion) zwar adäquat anzeigt, aber die höhere Plaquedicke (Scherkräfte) missachtet.

- Die Plaqueformation beeinflusst die Emboliegefahr.

- Die Messung der Querschnittsflächenreduktion in der CTA entspricht der duplexsonograhischen Stenosegraduierung über den stenosebedingten PSV-Anstieg nach dem Kontinuitätsgesetz am ehesten. 


\section{Korrespondenzadresse

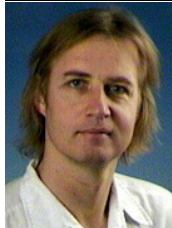 \\ Dr. W. Schäberle \\ Abteilung für Viszeral-, Thorax- und Gefäßchirurgie, Alb-Fils-Kliniken Eichertstr. 3, 73035 Göppin- gen, Deutschland wilhelm.schaeberle@af-k.de}

\section{Einhaltung ethischer Richtlinien}

Interessenkonflikt. W. Schäberle gibt an, dass kein Interessenkonflikt besteht.

\section{Für diesen Beitrag wurden von den Autoren keine} Studien an Menschen oder Tieren durchgeführt. Für die aufgeführten Studien gelten die jeweils dort angegebenen ethischen Richtlinien.

Open Access. Dieser Artikel wird unter der Creative Commons Namensnennung 4.0 International Lizenz veröffentlicht, welche die Nutzung, Vervielfältigung, Bearbeitung, Verbreitung und Wiedergabe in jeglichem Medium und Format erlaubt, sofern Sie den/die ursprünglichen Autor(en) und die Quelle ordnungsgemäß nennen, einen Link zur Creative Commons Lizenz beifügen und angeben, ob Änderungen vorgenommen wurden.

Die in diesem Artikel enthaltenen Bilder und sonstiges Drittmaterial unterliegen ebenfalls der genannten Creative Commons Lizenz, sofern sich aus der Abbildungslegende nichts anderes ergibt. Sofern das betreffende Material nicht unter der genannten Creative Commons Lizenz steht und die betreffende Handlung nicht nach gesetzlichen Vorschriften erlaubt ist, ist für die oben aufgeführten Weiterverwendungen des Materials die Einwilligung des jeweiligen Rechteinhabers einzuholen.

Weitere Details zur Lizenz entnehmen Sie bitte der Lizenzinformation auf http://creativecommons.org/ licenses/by/4.0/deed.de.

\section{Literatur}

1. AbuRhama AF, Robinson PA, Strickler DL, Alberts $S$ Young L (1998) Proposed new duplex classification for threshold stenosis used in various symptomatic and asymptomatic carotid endarterectomy trials. Ann Vasc Surg 12:349-358

2. Moneta GL, Edwards JM, Papanicolaou G, Hatsumaki T, Taylor LM Jr, Strandness DE Jr et al (1995) Screening for asymptomatic internal carotid artery stenosis: duplex criteria for discrimination $60 \%$ to $99 \%$ stenosis. J Vasc Surg 21(6):989-994

3. Grant EG, Duerinckx AJ, El Saden $S$ et al (1999) Doppler sonographic parameters for the detection of carotid stenosis. AJR Am J Roentgenol 172:1123-1129

4. Hood DB, Mattos MA, Mansour A et al (1996) Prospective evaluation of new duplex criteria to identify $70 \%$ internal carotid artery Stenosis. JVasc Surg 23:254-261

5. Huston J, James E, Brown RD Jr. et al (2000) Redefined duplex ultrasonographic criteria for the diagnosis of carotid artery stenosis. Mayo Clin Proc 75:1133-1140

6. Neale ML, Chambers JL, Kelly AT et al (1994) Reappraisal of duplex criteria to assess significant carotid stenosis with special reference to reports from the north American symptomatic carotid endarterectomy trial and the European carotid surgery trial. JVasc Surg 20:642-649

7. Carpenter JP, Lexa FJ, Davis JT(1995) Determination of sixty percent or greater carotid artery stenosis by duplex Doppler ultrasonography. J Vasc Surg 22:697-703

8. Faught $W E$, Mattos $M A$, van Bemmelen PS et al (1994) Color flow duplex scanning of carotid arteries: new velocity criteria based on receiver operator characteristic analysis for threshold stenosis used in the symptomatic and asymptomatic carotid trials. J Vasc Surg 19:818-828

9. Rothwell PM, Gibson RJ, Villagra R, Sellar R, Warlow CP (1998) The effect of angiographic technique and image quality on the reproducibility of measurement of carotid stenosis and assessment of plaque surface morphology. Clin Radiol 53:439-443

10. Waugh J, Sacharias N (1992) Arteriographic complications in the DSA era. Radiology 182:243-246

11. Hankey GJ, Warlow CP, Sellar RJ (1990) Cerebral angiographic risk in mild cerebrovascular disease Stroke 21:209-222

12. Naylor AR, Ricco JB, de Borst GJ, Debus $S$ et al (2018) Management of atherosclerotic carotid and vertebral artery disease: 2017 clinical practice guidelines of the European society for vascular surgery (ESVS). Eur J Vasc Endovasc Surg 55:3-81

13. Arous EJ, Simons JP, Flahive JM, Beck AW, Stone DH Hoel AW, Messina LM, Schanzer A (2015) National variation in preoperative imaging, carotid duplex ultrasound criteria, and threshold for surgery for asymptomatic carotid artery stenosis. J Vasc Surg 62:937-944

14. Arning C, Widder B, von Reutern GM et al (2010) Revision of DEGUM ultrasound criteria for grading internal carotid artery stenosis and transfer to NASCET measurement. Ultraschall in Med 31:251-257

15. Barlinn K, Rickmann H, Kitzler H et al (2018) Validation of multiparametric ultrasonography criteria with digital subtraction angiography in carotid artery disease: a prospective multicenter study. Ultraschall in Med 39:535-543

16. Moneta GL, Edwards JM, Chitwood RW, Taylor LM Jr, Lee RW, Cummings CA et al (1993) Correlation of north American symptomatic carotid endarterectomy trial (NASCET) angiography definition of $70 \%$ to $99 \%$ internal carotid artery stenosis with duplexscanning. JVasc Surg 17:152-159

17. Barnett HJM, Taylor DW, Eliasziw M et al (1998) Benefit of carotid endarterectomy in patients with symptomatic moderate or severe stenosis. NEng Med 339:1415-1425

18. European Carotid Surgery Trialists Collaborative Group (1998) Randomised trial of endarterectomy for recently symptomatic carotid stenosis: final results of the MRC European carotid surgery trial (ESCT).Lancet 351:1379-1387

19. von Reutern GM, Goertler MW, Bornstein NM et al (2012) Grading carotid stenosis using ultrasonic methods. Stroke 43:916-921

20. AbuRahma AF, Srivastava M, Stone PA, Mousa AY, Jain A, Dean LS, Keiffer T, Emmett M (2011) Critical appraisal of the carotid duplex consensus criteria in the diagnosis of carotid artery stenosis. J Vasc Surg 53:53-60
21. Jahromi AS, Ciná CS, Liu Y, Clase CM (2005) Sensitivity and specificity of color duplex ultrasound measurement in the estimation of internal carotid artery stenosis: a systemtic review and metaanalysis. JVasc Surg 41:962-972

22. Grant EG, Benson CB, Moneta GL, Alexandrow AV, Baker JD, Bluth El et al (2003) Carotid artery stenosis: grayscale and doppler ultrasound diagnosis-society of radiologists in ultrasound consensus conference. Ultrasound Q 19:190-198

23. Wardlaw JM, Chappell FM, Best JJK, Wartolowska K, Berry E (2006) Non-invasive imaging compared with intra-arterial angiography in the diagnosis of symptomatic carotid stenosis: a meta-analysis. Lancet 367:1503-1512

24. Gough MJ (2011) Preprocedual imaging strategies in symptomatic carotid artery stenosis. J Vasc Surg 54:1215-1218

25. Forjoe T, Asad Rahi M (2019) Systematic review of preoperative carotid duplex ultrasound compared with computed tomography carotid angiography for carotid endarterectomy. Ann R Coll Surg Engl 101(3):141-149. https://doi.org/10.1308/rcsann. 2019.0010

26. Schäberle W (2018) Sonographische Graduierung von Rezidivstenosen nach PTA und Stentimplantation. Gefäßchirurgie 23:465-482

27. AbuRahma AF, Richmond BK, Robinson PA et al (1995) Effect of contralateral severe stenosis or carotid occlusion on duplex criteria of ipsilateral stenosis: comparative study of various duplex parameters. J Vasc Surg 22:751-762

28. Schäberle W (2018) Ultrasonography in vascular diagnosis, 3. Aufl., S319-331

29. Romero J, Ackermann R, Dault N, Lev M (2005) Noninvasive evaluation of carotid artery stenosis: Indications, strategies and accuracy. Neuroimaging Clin N Am 15:351-365

30. Ohara T, Toyada K, Otsubo R, Nagatuka K, Kubota Y, Yasaka M, Naritomi H, Minematsu K (2008) Eccentric stenosis of the carotid artery associated with Ipsilateral cerebrovascular events. AJNR Am J Neuroradiol 29:1200-1203

31. Heath D, Smith P, Harris P, Winson M (1973) The atherosclerotic human carotid sinus. J Pathol 110:49-58

32. Solbert LA, Eggen DA (1971) Localization and sequence of development of atherosclerotic lesions in the carotid and vertebral arteries. Circulation 43:711-724

33. Eckstein HH, Winter R, Eichbaum M et al (2001) Grading of internal carotid artery stenosis: validation of doppler/duplex ultrasound criteria and angiography against endarterectomy specimen. Eur JVasc Endovasc Surg 21:301-310

34. Suwanwela N, Can U, Furie KL et al (1996) Carotid Doppler ultrasound criteria for internal carotid artery stenosis based on residual lumen diameter calculated from en bloc carotid endarterectomy specimens. Stroke 27:1965-1969

35. Widder B, Arnolds B, Drews S et al (1987) Bestimmung des Stenosierungsgrades bei Karotisstenosen: Ultraschall und i.v.DSA im Vergleich zum Operationsbefund. Ultraschall Med 8:82-86

36. Samarzija K, Milosevic P, Jurjevic Z, ErdeljacE (2018) Grading of carotid artery stenosis with computed tomography angiography: whether to use the narrowest diameter or the cross sectional area. Insights Imaging 9(4):527-534

37. Executive Committee for the Asymptomatic Carotid Atherosclerosis Study (1995) Endarterectomy for asymptomatic carotid artery stenosis. JAMA 273:1421-1428 
38. Halliday A, Mansfield A, Marro J et al (2004) Prevention of disabling and fatal strokes by successful carotid endarterectomy in patients without recent neurological symptoms: randomized controlled trial. Lancet 363:1491-1502

39. Zhang Z, Berg M, Ikonen A, Könönen $M$, Kälviänen $\mathrm{R}$, Manninen $\mathrm{H}$, Vanninen $\mathrm{R}$ (2005) Carotid stenosis degree in CT angiography: assessment based on luminal area versus luminal diameter measurements. Eur Radiol 15:2359-2365

40. Carnicelli AP, Stone JJ, Doyle A, Chowdhry AK, Mix D, Ellis J, Gillespie DL, Chandra A (2013) Crosssectional area for the calculation of carotid artery stenosis on computed tomographic angiography. JVasc Surg 58:659-665

41. Tambasco M, Steinman DA (2003) Path-dependent hemodynamics of the stenosed carotid bifurcation. Ann Biomed Eng 31:1054-1502

42. Nighoghossian N, Derex L, Douek P (2005) The vulnerable carotid artery plaque. Current imaging methods and new perspectives. Stroke 36:2764-2772
43. Zarins CK, Gidens DP, Bharadvaj BK et al (1983) Carotid bifurcation atherosclerosis. Quantitative correlation of plaque localization with flow velocity profiles and wall shear stress. Circ Res 53:502-514

44. Cheng C, Tempel D, van Haperen R et al (2006) Atherosclerotic lesion size and vulnerability are determined by patterns of fluid shear stress. Circulation 113:2744-2753

45. Steinman DA, Poepping TL, Tambasco $M$ et al (2000) Flow patterns at the stenosed carotid bifurcations: Effect of concentric versus eccentric stenosis. Ann Biomed Eng 28:415-423

46. Hoogi A, Adan D, Hoffman A (2011) Carotid plaque vulnerability: quantification of neovascularization on contrast-enhanced ultrasound with histopathologie correlation. AJR Am J Roentgenol 96:431-436

47. Staub D (2015) Arteriosclerotic plaque neovascularization and inflammation-is there a link? Vasa 44:163-165

48. Staub D, Pavoti S, Schinkel AF (2011) Correlation of carotid artery atherosclerotic lesion echogenicity and severity at standard US with intraplaque neovascularization detected at contrast-enhanced US. Radiology 258:618-626

\section{Praxis-Finanzrechner für Ärzte Neues Tool auf SpringerMedizin.de}

Wie rentabel läuft meine Praxis? Wie stehe ich im Vergleich zu meinen Kollegen im Bundesland? Welche Anschaffungen kann ich mir leisten? Antworten auf diese betriebswirtschaftlichen Fragen liefert künftig ein Tool auf SpringerMedizin.de, indem es aktuelle Versorgungs- und Abrechnungsdaten sowie Analysedaten deutscher Gesundheitsunternehmen zu Grunde legt.

\section{Mit den kostenfreien Praxis-Finanzrechnern können sich vor} allem in der Praxis tätigen Ärzte, die sich oft auch unternehmerischen Herausforderungen stellen müssen, interaktiv mit den Themen Controlling und Benchmarking auseinandersetzen:

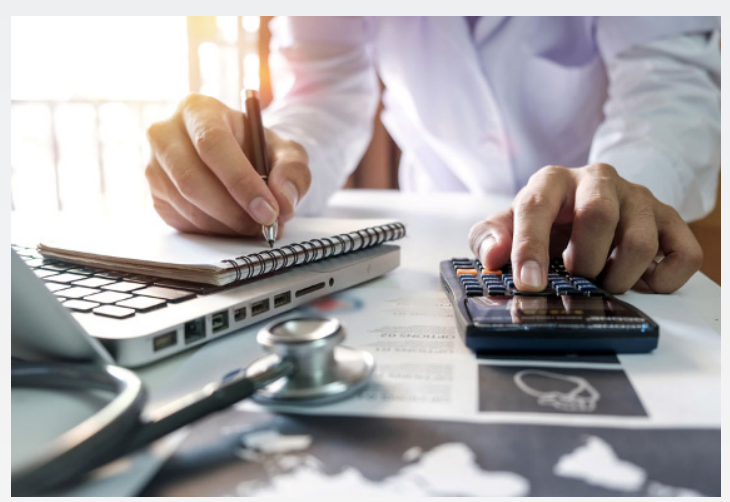

(c) mrmohock/stock.adobe.com
Mit dem Controlling-Assistenten behalten Sie die wichtigsten wirtschaftlichen Praxiskennzahlen immer im Auge. $>$ Mit dem Tool Benchmark können Sie Ihre Praxis mit anderen Praxen vergleichen.

$>$ Ihren Praxisstandort - ebenfalls ein wichtiger Faktor für den Erfolg - analysieren Sie mit Standortcheck aus verschiedenen Perspektiven.

> Mit dem Investitionsassistenten können sie sehen, ob und wann sich eine Geräteinvestition amortisiert und Sie bekommen auch Anhaltspunkte, welche Einnahmen Sie zu erwarten haben.

Die Praxis-Tools sind ein externes Angebot von Rebmann Research, Partner von SpringerMedizin.de. Sie finden die Praxis-Finanzrechner auf SpringerMedizin.de unter dem Navigationspunkt "Mehr".

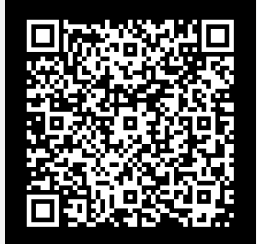

